\title{
CRIMEN COMO FACTOR DE CAMBIO URBANO: UN ESTUDIO SOBRE LAS POLÍTICAS DE SEGURIDAD PÚBLICA EN RÍO DE JANEIRO
}

\author{
Livio Silva de Oliveira ${ }^{1}$
}

\begin{abstract}
Resumen: La seguridad pública ha ganado cada vez más espacio en la agenda de la Administración Pública en Brasil y exigió nuevas formas de enfrentamiento contra el crimen, convirtiéndose en tema de interés sociológico. Este trabajo tiene como objetivo analizar el proceso de desarrollo e implementación de las Unidades de Policía Pacificadora (UPP) en la ciudad de Río de Janeiro. Para entender el formato de este modelo de policía se hizo un esfuerzo histórico y sociológico de los procesos de urbanización de Río de Janeiro y de criminalización de las personas marginadas de esa ciudad. Desde el concepto de rotulación, en la teoría de la desviación, defendida por Taylor, Walton y Young, de la Escuela Británica de Criminología Crítica; se analizaron las contradicciones y perspectivas sobre la reacción social contra los delitos y su correlación con los factores sociales.
\end{abstract}

Palabras clave: Territorio; UPP; Criminalización.

\section{Crime as a factor of urban change: a study on public security policies in Rio de Janeiro}

\begin{abstract}
Public safety has gained more and more space on the agenda of the Public Administration in Brazil and demanded new ways of fighting against crime, becoming a subject of sociological interest. This work aims to analyze the process of development and implementation of the Pacifying Police Units (UPP) in the city of Rio de Janeiro. To understand the format of this model police became a historical and sociological effort urbanization's process of Rio de Janeiro and criminalization of marginalized people of that city. Since the concept of labeling, in the theory of deviance, defended by Taylor, Walton and Young, British School of Critical Criminology; contradictions and perspectives on the social reaction against crime and its correlation with social factors were analyzed.
\end{abstract}

Keywords: Territory; UPP; Criminalization.

\section{Introducción}

Las explicaciones para el fenómeno de la violencia en la sociedad son diversas. Pasan de círculos de conversación en los bares, cenas y reuniones familiares, en su mayoría basados en el sentido común y la experimentación pura de prejuicios sociales que no son exactamente reflexivo, por así decirlo; a la academia (que, en cierto modo, con un aura de legitimidad como si estuviera produciendo verdades a través de sus discursos de neutralidad aparente, que es justo lo contrario de la ciencia), esferas institucionales del Estado y los medios de

\footnotetext{
1 Doutorando em Sociologia pela Universidade Federal do Rio Grande do Sul. Contato: livioxt@gmail.com
} 
comunicación. El hecho es que parece que la violencia es un fenómeno social universal y generalizado que afecta a todos los individuos de una sociedad.

Sin embargo, hay matices en estas interacciones sociales. Ciertos tipos y grupos sociales históricamente han sido clasificados como "clases peligrosas". La composición de este cuadro presenta factores sociales, culturales, históricos, étnico-raciales, económicos, de género, filosóficos, religiosos, jurídicos y políticos, que pueden ser yuxtapuestos en un individuo que pertenece a un grupo social determinado. Para comprender el proceso de criminalización de ciertos grupos, es necesario analizar la reacción y/o aceptación social de la desviación y la carrera desviada. Por consiguiente, la desviación se articula con el concepto de rotulación. La definición de un problema sociológico haz mitos construidos a través del sentido común sobre la violencia serán rotos. Mitos que pueden guiar interacciones sociales y relaciones de poder asimétricas, profundizando de las desigualdades relacionales.

El objeto empírico para analizar y demostrar cómo la teoría de la desviación se aplica en realidad es la política de seguridad pública del gobierno estadual ${ }^{2}$ de Río de Janeiro, las Unidades de Policía Pacificadora, conocidas por el acrónimo UPP. El modelo implementado a partir de 2008 tuvo como uno de sus objetivos la integración de zonas marginalizadas y criminalizadas a lo tejido urbano de la ciudad de Río de Janeiro, capital del estado homónimo. Pero, algunos conflictos surgen de esta interacción entre poder estatal y las poblaciones de estos territorios, que se suman a un histórico de estigmatización social y de violencia. Estos factores son problematizados en este texto para identificar cuáles son las posibles relaciones entre la delincuencia y la división espacial de Río de Janeiro, y entender los matices del proceso de implementación de las UPP en este contexto.

\section{Desviación y rotulación: enfoques teóricos para la comprensión de los crímenes violentos}

La teoría de la desviación indica otras situaciones relacionales que causan ciertas acciones: la aceptación y la reacción a las desviaciones sociales (TAYLOR WALTON, YOUNG, 1990). Sobre la base de esta valoración se desarrollan mecanismos de control social de las desviaciones. En el campo científico, Criminología y Sociología rechazan las nociones positivistas y explicaciones genéticas para el crimen y la desviación (Ibíd. p. 157). La reacción social se lleva a cabo a través de la aceptación del público o el rechazo de la acción. No es el acto en sí, sino cómo, por qué y quién lo practica será decisivo. Para analizar este

\footnotetext{
${ }^{2}$ Los gobiernos estaduales tienen equivalencia con los gobiernos provinciales de Argentina en relación división territorial de las tres esferas de poder.
} 
proceso, los autores se refieren al concepto de rotulación. Preguntas como "desviados para quién?" y "desviados en comparación con qué?" denotan la asimetría en la relación de poder contenida en esto concepto. Por lo tanto, el proceso de categorización de quién es lo desviado o no en una determinada sociedad presenta un sesgo político muy marcado, que también indica un proceso con fuertes connotaciones morales a través de una represión normalizada por las elites gobernantes (Ibídem. p.186). En síntesis, la desviación y la rotulación son indicadores para entender las relaciones de poder de una sociedad.

Las desviaciones son acciones deliberadas de violación del código moral y / o el código legal de la sociedad, por diversas razones. Pueden ser de órdenes primarias y secundarias, afirman Taylor, Walton y Young. La desviación primaria apunta para lo cambio de conducta como respuesta marginal a la reacción social hacia los que practican o han querido desviar. La desviación secundaria se caracteriza cuando las causas de la desviación retroceden y dan lugar a reacciones de desprecio, de desaprobación y de rotulación de la sociedad (TAYLOR WALTON, YOUNG, 1990; p. 167-168). Para los autores, la desviación debe ser pública para que haya el proceso de rotulación, ya que la publicidad del acto es lo que definirá lo éxito de su empleo o no, es decir, cuando se transforma un rasgo inseparable de la identidad social del individuo.

La desviación apunta para desigualdades sociales más amplias entre poder y autoridad. En este sentido, el proceso de rotulación también puede indicar la agresión como defensa contra las pérdidas materiales, simbólicas, legales y emocionales. Las personas cometen actos desviados debido a acontecimientos y circunstancias de su vida, independientemente de las etiquetas que se ponen en ellos, pero ni todos son enviados a los valores despectivos en la sociedad, defienden Taylor, Walton y Young. Es cierto que aquellas personas consideradas desviadas surgen con frecuencia en la oposición de ciertos grupos de la sociedad, lo que hace que se conviertan en actores sociales (TAYLOR WALTON, YOUNG, 1990; p. 173) y, por lo tanto, pueden ser considerados delincuentes en dos maneras para los autores: adquiridos y asignado. El primer caso se refiere a aquellas personas sujetas a control social. El segundo caso se refiere a aquellos que pueden organizarse para cambiar los valores sociales estructuralmente y liberarse de los estigmas en sus "defectos". En otras palabras, lo desviado no es un mero producto de la sociedad, pero alguien que puede rebelarse contra ella, porque la sociedad está en constante cambio. Por lo tanto, la complejidad en los procesos de control social y de rotulación indica cuales son los tipos de intereses políticos que están en juego y 
cuales son los conflictos sociales que llevan a esta dinámica, la estructuración de un conjunto de leyes y reglamentos que interviene directamente en el proceso de criminalización.

La delincuencia, Taylor, Walton y Young, es una de las etapas de la conducta criminal. Las desviaciones pueden ser motivadas por un sistema de creencias, sean ellas verdaderas o falsas, que conducen a actos desviados, no siendo por tanto, fenómenos distintos. Además, critican las falsas dicotomías en la teoría de la desviación, por ejemplo, la eliminación de la distinción entre el individuo y la sociedad y que el fin de la propiedad privada tendría como consecuencia el fin del robo (TAYLOR WALTON, YOUNG, 1990; p. 191). Ellos resumen las contradicciones sociales a través de la flexibilidad moral de los delincuentes en una sociedad de conductas delictivas. El robo de una gran empresa tendría importantes consecuencias porque el seguro cubriría los daños, por ejemplo. El sistema normativo de una sociedad se caracteriza por la flexibilidad y la persona puede evitar la culpabilidad moral de su acción delictiva. En este sentido, la subcultura delictiva es una imagen equivocada por tener como referencial un sistema de valores de la clase media, porque la sociedad no consiste solamente por esta clase. El delincuente no es un extraño para el cuerpo de la sociedad, teniendo la posibilidad de una conducta criminal sea una acentuación de los valores dominantes. La propia idea de delincuentes transitorios o no - las diferencias entre adultos y la etapa juvenil de la vida (Ibíd. p. 197) - es rechazada por los autores, ya que es una distinción artificial.

Desde esta perspectiva, Taylor, Walton y Young afirman que la sociedad no es sólo un conjunto de normas y, si, que hay una relación social entre los individuos. La internalización de reglas sociales no transforma el individuo en un autómata porque las reglas son interpretativas. Además, las normas cotidianas no son inmutables y se caracterizan por su ambigüedad, lo que evidencia la defensa de los autores de que la sociedad está en constante cambio. Así, no hay manera de afirmar la existencia de un individuo desviado abstracto porque le falta base material para su verificación en la sociedad.

\section{UPP: Integración o segregación?}

En diciembre de 2008, el gobierno del estado de Río de Janeiro implementó su primera Unidad de Policía Pacificadora, presentada como una nueva forma de actuación policial y la seguridad pública en los territorios marginalizados y criminalizados de Río de 
Janeiro, conocidos por el término favela. Esto modelo de seguridad pública es popularmente llamado por su acrónimo: UPP. El proyecto-piloto fue desarrollado en morro Santa Marta, barrio de Botafogo, ubicado en la zona sur de la ciudad. A pesar de la novedad de la idea de la UPP, vale la pena señalar que otros experimentos se han tratado en esta dirección en las favelas cariocas, como los Postos de Policiamento Comunitário (PPC) y los Grupamentos de Policiamento em Áreas Especiais (GPAE), que no pudo cambiar la interacción entre La policía y los residentes, históricamente marcada por conflictos que podrían llegar a actos violentos, (CUNHA y MELLO, 2011. p. 373). Otro punto a destacar en la UPP es la idea de la paz en lugar de la noción de guerra.

Actualmente, el Río de Janeiro cuenta con 38 UPP, en las que existe un efectivo 9.543 de policiales, según el sitio web oficial de la Secretaría de Estado de Seguridad Pública (SESEG). Las fuerzas policiales son parte de la ocupación, el principio - en algunos casos, incluso las fuerzas federales - y un comandante militar sería el encargado de la entrada de ese estado en el vacío dejado por el crimen, ya que su objetivo principal es la ocupación del territorio por parten de la Administración Pública. Por otra parte, las UPP vienen con una propuesta de policías recién graduados para una policía de aproximación con los residentes. Sobre la participación de las fuerzas federales en el proceso de aplicación de la UPP, especialmente los de élite del ejército tropas (paracaidistas y policía del Ejército) y de la Marina (fusileros navales), que tuvo lugar en ciertas partes de la ciudad considerados por las autoridades y la opinión pública como "más críticos", los complejos de favelas en la zona norte de Río de Janeiro, efectivamente: Complexo do Alemão (2010) y Complexo da Maré (2013). En el momento de la ocupación de las favelas de Complexo do Alemão (que abarca los barrios de Olaria, Penha, Ramos, Bonsucesso y Inhaúma) fueron difundidos por los medios de comunicación con titulares como "día histórico", "El Estado ha derrotado los narcos" entre otras.

Las UPP también tienen proyectos sociales dentro de su programa, que son implementados por la alcaldía de Río de Janeiro y coordinados por el Instituto Pereira Passos (IPP), que se llaman UPP Social: " A UPP Social é a estratégia de promoção de integração urbana, social e econômica das áreas da cidade beneficiadas por Unidades de Polícia 
Pacificadora (UPPS)"3. Además, el programa UPP Social tiene un acuerdo de cooperación firmado entre la municipalidad y la ONU-Hábitat, el programa de las Naciones Unidas para los asentamientos humanos. Otras asociaciones para las UPP son del sector privado tales como bancos y otras ramas de negocio. Un punto que hacerse es que el programa UPP Social está en 25 territorios (que pueden tener más de una UPP), sin contar las zonas que no han sido implementadas la política de la UPP, según el sitio web oficial del propio programa.

Las UPP presentan una planificación más sistemática y se definida, en relación con los delitos violentos. Sin embargo, el crimen es un fenómeno más complejo y más amplio. Problematizar, en este sentido, el concepto de crimen y comprender por qué el enfoque de seguridad pública en la ciudad de Río de Janeiro son los territorios marginalizados sirven de recorte para analizar las desigualdades en la sociedad. Esta descripción de la realidad material de Río de Janeiro será articulada con la teoría de la reacción social a desviación a través del proceso de rotulación, desde la perspectiva de la Criminología Crítica, para transformar la política de seguridad pública en un problema sociológico.

El proceso de construcción social e histórica de las favelas de Río de Janeiro pasó por varias fases. El término favela fue introducido en Río de Janeiro por los veteranos de la Guerra de Canudos. En 1897, al final del conflicto armado, ocurrido en estado de Bahia, noreste de Brasil, cerca de 10.000 veteranos fueron a ciudad, entonces capital del país, cobrar la promesa hecha por el gobierno federal a ellos: la vivienda. Ex combatientes permanecían acampados en las afueras del Ministerio de la Guerra, por la calle Barão de São Félix, en el centro. La promesa no se cumplió, y los campistas han asentado arribaran para un territorio cercano del Ministerio, que se hizo conocido como Morro da Favela (SILVA DE OLIVEIRA, 2014. p. 56). No ha tardado para que El Morro da Favela fuese visto por la élite y las autoridades locales como un problema social, así como una molestia estética y de salud por su aglomerado de casas populares y sus conjuntos de viviendas colectivas conocidos por cortiço. Estos valores despectivos también se refieren a la idea de la criminalización. Actualmente, esta zona se llama Morro da Providencia y tiene una UPP.

Otra fase de las favelas de Río de Janeiro son las remociones. A pesar de la acción de quitar conglomerados de viviendas ser una práctica recurrente en la historia de la ciudad - el

\footnotetext{
${ }^{3}$ Libre traducción: "La UPP Social es la estrategia de promoción de integración urbana, social y económico de las áreas de la ciudad que han sido beneficiadas por las Unidades de Policía Pacificadora (UPP)".
} 
ejemplo más clásico de esta acción fue la reforma urbana promovida por Pereira Passos, nombrado alcalde de la entonces capital federal (1902-1906), que se hizo popularmente conocido como "bota-abaixo", que tenía como objetivo retirar forzosamente los cortiços del centro de la ciudad, incluso con lo derrocamiento de las casas y empleo de la violencia, caracterizando los contornos higienistas barnizados en un discurso de la modernización de las autoridades e de las elites - las remociones ganaron más fuerza en la década de 1960, bajo la dictadura cívico-militar en Brasil. Las favelas fueron removidas a regiones alejadas del centro y sur de la ciudad, a valorar las dos últimas áreas. La violencia utilizada en las remociones se ha tornado más fuerte en esto periodo, lo que profundizó el conflicto en la relación entre el Estado y la población marginalizada, y el riesgo constante de ser removido (BRUM, 2013). Con la democratización del país, en 1988, el riesgo de remociones ha ido disminuyendo. Sin embargo, la transición política no fue el único factor que se ha convertido en una barrera para la remoción de las poblaciones de territorios marginalizados y criminalizados.

Otro factor que contribuyo para reducir los riesgos de remoción fue el crecimiento del crimen organizado en las favelas. La ciudad de Río de Janeiro entre los años 1970 y 1980 se convierte en un polo consumidor de drogas, en especial la cocaína (GRILLO \& MISSE, 2014). Esto fenómeno aumentó la renta de las pandillas locales, llevándolas a comprar armas de guerra para defender sus posiciones de ventas al por menor de drogas y ejercer el control social informal e ilegal sobre las poblaciones marginalizadas, al paso de los narcotraficantes competieren con el Estado (SILVA DE OLIVEIRA, 2014. p. 71). Los años 1983 y 1984 se pueden considerar el plazo de la consolidación del mercado de la cocaína en la ciudad, la proximidad de grandes grupos de narcotráfico internacional con narcotraficantes locales (SILVA et al, 2008. p. 20). Este cambio se tradujo sustancialmente pasando de paradigma "marihuana 38" a "la cocaína AR-15", en referencia a las armas utilizadas por pandillas que ejercieron el poder en zonas marginalizadas y criminalizadas (SILVA et al, 2008). Con el creciente potencial bélico de narcotraficantes de Río de Janeiro, la incursión de la policía en las favelas de la ciudad se ha convertido en una actividad de riesgo, lo que dificultó las acciones de remoción. Pero, los servicios de la administración pública, tales como saneamiento, electricidad, entre otros, también fueron obstaculizados y / o hechos de forma precaria e insatisfactoria.

Desde los años 1980, la violencia se ha cambiado más visible en las favelas de Río de Janeiro. Acciones de seguridad pública en estas áreas se caracterizan por una dinámica 
discontinuada. Además, la discrepancia entre sus aplicaciones fue evidente. El primer gobierno estadual de Leonel Brizola (1983-1987) estuvo marcado por la vigilancia de la humanización de las patrullas en las zonas marginalizadas. La ideología de los derechos humanos en la policía de Río de Janeiro siguió en las políticas de seguridad pública del segundo gobierno de Leonel Brizola (1991-1994), o sea, este diseño fue el sello distintivo de la administración estatal de la seguridad pública en esos períodos (MIRANDA, 2015 . p. 4). Brizola ha sido criticado por las elites y las clases medias de la ciudad para tomar un tratamiento "blando con los delincuentes", lo que estimularía la acción delictiva (SILVA DE OLIVEIRA, 2014. p. 68), mostrando, que en sentido común, los derechos civiles son entendidos como un obstáculo para el control social.

En oposición a la idea de humanización, otro ejemplo se utiliza para ilustrar el proceso histórico de discontinuidad de las políticas de seguridad pública en Río de Janeiro fue la gratificación pecuniaria a los policiales por "actos de valentía". En 1995, el entonces gobernador del estado, Marcello Alencar (1995-1999), ha creado por decreto la dicha gratificación para aquellos agentes que participasen en las operaciones principales. Sin embargo, después de tres años de este premio, las cifras de homicidios en el estado no han disminuido, resalte la polémica de la bonificación, que se conoció como la gratificación faroeste, en alusión a las películas de western estadunidenses. La gratificación faroeste es un ejemplo de la recrudescencia y de la militarización de la seguridad pública, dado que, a época, el Secretario de Seguridad Pública de Río de Janeiro era el General de Ejército Nilton Cerqueira; que tenía como objetivo prioritario para su rigidez las personas más pobres y “os efeitos desta política foram o incremento da mortalidade entre os policiais e a constatação de sua mira certeira, pois em 'confronto' com os supostos 'bandidos', conseguem sempre atirar para matar" " (CANO, 2007 apud MIRANDA, 2015, p. 5). En ese sentido, no existe una base teórica para apoyar la eficacia de este modelo reactivo para reducir el crimen, aunque haya un fuerte apoyo de la opinión pública.

El crimen organizado fue uno de los factores de control social de los territorios marginalizados de Río de Janeiro y la no remoción de las poblaciones de sus lugares de origen. Con esto, la rotulación de lugares peligrosos fue empleada con más fuerza por la élite de la ciudad en referencia a las favelas a partir de la década de 1980, no sólo como lugares

\footnotetext{
${ }^{4}$ Libre traducción: "los efectos de esta política fueron el aumento de la mortalidad entre la policía y la verificación de su mira infalible, porque en 'confrontación' con los supuestos criminales, siempre logran disparar a matar".
} 
incivilidad, la violencia, la falta de higiene, el retraso, en fin, todo tipo de adjetivo despectivo otorgan a residentes de los territorios; se añadió la idea de "guerra" en la etiqueta. El enfrentamiento armado entre narcotraficantes y policías ocasionó un sentimiento difuso de miedo e inseguridad entre la población y profundizó el estigma social sobre los vivientes de las favelas. La UPP surgió como la permanencia del Estado en las favelas, donde él llegaba de manera efectiva a través de su policía, el brazo del estado que siempre ha tenido acceso a estos territorios, después de varios intentos. En este sentido, la "corrección" de las desviaciones de aquella población marginalizada y crimenalizable por la presencia del Estado coercitivo parece tener una connotación mesiánica, es decir, las favelas podrían ser parte de la ciudad formal. Así, las UPP presentan otro carácter funcional: convertir la ciudad real en la ciudad formal.

Los territorios de vivienda y convivir populares presentan este aspecto de la división urbana entre lo real y lo formal a nivel global. En el caso de la ciudad de Río de Janeiro, la división se materializa por la categoría favela. Esta categoría en su perspectiva sociológica indica componentes despectivos, por una élite dominante, que se añaden a las ya presentadas en el aspecto humano de sus poblaciones. Prejuicios raciales (negros), orígenes (generalmente nordestinos ${ }^{5}$ ) son partes de la construcción social de la desviación de las poblaciones marginadas en Río de Janeiro, así como los factores sociales, económicos y políticos. Por lo tanto, el proceso de rotulación presenta también su estética física y simbólica, a partir de las normas sociales de belleza y del origen que transforman determinados cuerpos socialmente adherentes a estos rótulos despectivos.

La expulsión y / o la invisibilidad de los narcotraficantes en las favelas de Río de Janeiro por sí solas no serían capaces de promover la pacificación en estos territorios. E1 derecho a la vivienda, consolidado en la re-democratización de Brasil por la Constitución Federal de 1988 fue uno de los factores que hicieron las remociones más difíciles. La implementación del modelo UPP tenía como uno de los aspectos positivos la posibilidad de regulación de la tierra, o sea, los residentes de las favelas pueden dar legalidad a sus hogares, suelos, etc. (CUNHA \& MELO, 2011; OLIVEIRA \& NUÑEZ, 2012). Sin embargo, la entrada del Estado a través de la recaudación de impuestos aparece como una novedad en la vida cotidiana de las favelas y presenta el miedo de la población local de una expulsión blanca por la imposibilidad de hacer frente a estos nuevos costos (OLIVEIRA \& NÚÑEZ,

\footnotetext{
${ }^{5}$ Término referente a los individuos venidos del nordeste de Brasil.
} 
2012. p. 177). Hay, entonces, un modelo de formalización de ciudades que llegan a los territorios que reciben la UPP, que implica cuestiones de ajustes socioeconómicos. En otras palabras, los impactos sociales de este proceso pueden ser la gentrificación de la población marginalizada, que puede verse obligada a emigrar a otras áreas periféricas consideradas descalificadas por las élites, permaneciendo, así, dentro del espectro de la rotulación por la falta de movilidad social de esta población, materializado por su lugar de residencia y convivencia.

Las UPP, por lo tanto, parecen hacer parte de un proyecto más grande que una política de seguridad pública. Las diferentes formas en que se implementan estas unidades dan el tamaño de la división de la ciudad de Río de Janeiro y sus objetivos más inmediatos. Mientras que en las favelas de la Zona Sur, la zona principal de la ciudad, el proceso fue lo más discreto y pacífico posible, es decir, sin intercambio de disparos de armas de fuego. Ya en el zona norte, las zonas suburbanas y periféricas, la violencia visual y física - hombres armados, vehículos blindados y fuerzas federales - fue la tónica, con la excepción del barrio de Tijuca, que es compuesto en su mayoría por la clase media y la clase media alta.

Los aspectos económico y estratégico de la ciudad son considerados en esto contexto. La zona sur de Río de Janeiro tiene potenciales de bienes inmobiliarios y turístico, impulsado por los mega-eventos (Copa del Mundo de 2014 y los Juegos Olímpicos en 2016 - que abarca también el barrio de Tijuca por cuenta del llamado corredor olímpico de Maracaná), lo que demanda una sensación de seguridad para dar más comodidad y que no sea agresivo a los ojos. Mientras que las UPP más periféricas tienen dos funciones: los que están cerca del puerto (en el centro de Río de Janeiro) y en el camino desde el aeropuerto internacional Tom Jobim (ubicado en la Base Aérea de Galeão a localidad nombrada Ilha do Governador, al norte de la capital del estado), que pasa por el complejo de favelas de la Maré, a través de la autopista urbana línea roja y por la avenida Brasil, tienen roles estratégicos para mantener la seguridad de los visitantes y de negocios. Las otras UPP aparecen con función simbólica al ser zonas consolidadas en el imaginario social como violentas y de consumo considerable de drogas, como en el complejo de favelas de Jacarezinho, también en el norte de la ciudad. Por tanto, la rotulación está presente en las formas de implementación y desarrollo de las UPP universalmente, a pesar de las distinciones de sus objetivos para cada zona de la ciudad.

La militarización de los territorios por parte de la UPP es confrontante con la perspectiva de los derechos humanos. Aunque la policía local pueda garantizar el acceso a un 
cierto nivel de servicios del Estado a las zonas que fueron áreas de conflictos armados, tales como saneamiento, electricidad, etc., para traer beneficios materiales a las localidades, la garantía de los derechos de ciudadanía y de la participación social es cuestionable, uno de los indicios son las recurrentes quejas de abusos y crímenes cometidos por la policía (CANO, 2012; MOURÃO, 2013). El evento más emblemático en este sentido fue la desaparición del albañil Amarildo Gomes da Silva, en la favela de Rocinha, que tiene una UPP, sirvió como catalizador para la crítica del modelo. Según los testigos, Amarildo fue retirado de su casa por la policía y conducido, sin una orden judicial, a la sede de la UPP local y después de eso no fue más visto. El caso Amarildo señaló el creciente número de desaparecidos en los territorios donde se implementó ese modelo de seguridad pública desde que se abrió. La muerte de Eduardo de Jesús, de apenas diez años de edad, en el Complexo do Alemão, en abril de 2015, también señala los posibles resultados de la militarización de la seguridad pública, ya que el disparo que golpeó la cabeza del niño recibió salió del fusil de un policía que estaba operando en la localidad. La permanencia de la Policía indica el cambio morfológico de las relaciones sociales, puede ser un paso hacia el Estado cada vez más presente en los territorios marginalizados y con ello transformar la interpretación de la población local sobre la policía, marcada por la desconfianza mutua, o profundizar la relación ya deshilachada entre estos dos actores. Los matices de esta policía militarizada y permanente en materia de derechos humanos señalan el conflicto histórico entre marginalizados y las fuerzas del Estado. Sin embargo, la reflexión sobre cómo es la intervención del Estado en estas áreas tiene como un componente de la falta de estos derechos, sea por la pérdida de la relación de alteridad por la presencia del "movimiento" o de la fuerza policial. La violencia ilegal o la institucional pueden producir el mismo efecto en ese escenario.

La formalización de la ciudad real tiene aspectos estandarizados, que remite a lo noreconocimiento de los diferentes factores de sociabilidad. El tipo de organización social en las favelas es interpretado como desorden por autoridades y élites Río de Janeiro. Pero hay un control social del espacio, aunque sea de manera ilegal por bandas de narcotraficantes u por otros atores internos tales como la asociación de residentes. Es decir, otro tipo de sociedad dentro de la ciudad formal, hasta el punto de expresión poder paralelo se ha hecho populares en referencia a los narcotraficantes en las favelas de Río de Janeiro. Ahora, si no hubiera una capacidad organizativa mínima en estos territorios por los que viven locales, la interacción social sería poco probable. Esta perspectiva destaca la permanencia de un desprecio histórico 
y cultural de las élites políticas y económicas de Río de Janeiro, en relación con los favelados, que legitima las acciones de control social a través del uso de la fuerza. Por otra parte, indica el lenguaje por la cual se dio la gramática de los conflictos locales y su resolución o no, y, por lo tanto, el tipo de actos que se consideran desviaciones y crímenes dentro de este microcosmos urbano. Además, el hecho de no estar formalmente en la ciudad no significa estar aparten de la urbes, ya que la circulación entre la ciudad formal y la ciudad real se lleva a cabo de diversas maneras: el mercado de trabajo, medios de comunicación, entre otros, que se centran en el mundo de las percepciones, valores y acciones de los individuos. En otras palabras, no es sólo una división física de la ciudad, sino también una división en el sentido simbólico.

Por último, las UPP llevan en sí una incógnita: son incluyentes o excluyentes? La pregunta es compleja, ya que no es algo lineal. Por un lado, la necesidad de una actuación policial diferenciada ya refuerza el estigma social de marginados del tema de la delincuencia violenta, y la permanencia de que la policía especial por lo que estas comunidades se extienden en el tiempo puede delimitar, formal y legalmente, los límites de ciudad, siendo punto de crítica por no promover y garantizar los derechos de ciudadanía de manera universal. La fracción del derecho más evidente en las UPP fue de los consumidores. Por otro lado, el Estado no puede ser negligente a zonas urbanas históricamente abandonadas, que se tornaron áreas de conflictos armados. Además, las acciones no podrían seguir lo mismo estilo de reacción violenta, lo que ha demandado una alternativa de policía en las favelas.

La ecuación, en este sentido, no es la más fácil, lo que da la dimensión de la complejidad del crimen. No hay sociedad sin crimen, al igual que no existen barreras sociales para que ocurra. Así, el crimen es un fenómeno social extra e inter clases. El proceso de rotulación a través de la reacción social a la desviación es parte de un catálogo de sospechosos ideales a través de un gran crimen que luchar, un delito público, por así decirlo; en el caso: el tráfico de drogas. Aunque este delito sea patrimonial, las proporciones que ganó en Río de Janeiro son más altas debido a la cantidad de muertes violentas. Esto no es exclusividad de la ciudad o de Brasil, es un fenómeno verificado en una escala global. Por lo tanto, las UPP traen preguntas más profundas que algo cerrado a seguridad pública en sí misma. Por cierto, las UPP nos dan más preguntas que respuestas. Ellos reflejan las incertidumbres y los valores de una sociedad desigual y sus relaciones de poder, y en ese sentido, con el proceso de rotulación aún pueden producir criminales culpados hasta que se demuestre lo contrario, 
invirtiendo el precepto legal de presunción de inocencia, apuntando también un marco de injusticia.

\section{Conclusión}

A partir de la utilización de la teoría de la reacción social a la desviación y el concepto de rotulación se realizaron análisis del desarrollo histórico de las UPP y el proceso de criminalización. Se ha verificado que este modelo no es exactamente una novedad en el sentido de permanencia de las fuerzas policiales en los territorios marginalizados y criminalizáveis en la ciudad de Río de Janeiro, pero su forma y contenido difiere de los intentos anteriores. Las UPP ganaron contornos mesiánicos a través de los discursos construidos por los medios de comunicación, por las elites y las autoridades de Río de Janeiro. Sin embargo, esto modelo muestra signos de desgaste debido a algunos de los lugares que fueron implementadas las unidades volvieron a registrar los conflictos armados.

La construcción social de las clases peligrosas fue intrínseca a la construcción espacial de la ciudad de Río de Janeiro. La percepción de las favelas como símbolo de retraso, contrario a la modernidad del plan europeo, y sus habitantes eran incivilizados, lo que legitimó acciones violentas contra esta población marginalizada. Las remociones eran una realidad constante, lo que denota la desigualdad social que ha reflejado la falta de reconocimiento de los grupos sociales de los ciudadanos considerados desviados. Por otra parte, el estado de abandono en relación a los territorios marginalizados contribuyó a las formas locales de control social. En este sentido, las bandas de narcotraficantes jugaron un papel clave, al punto, desde la década de 1980, estos grupos criminales fueron capaces de competir con el Estado a través de su fuerza militar. Por lo tanto, el tráfico ilícito de drogas se ha convertido en un problema de Estado de Río de Janeiro, cuando el poder de los grupos criminales alcanzó una meseta capaz de subvertir el orden estatal.

Por fin, hay una división entre la ciudad real y la ciudad formal que es uno de los factores determinantes para la UPP para ser un modelo. La criminalización de los territorios marginalizados legítima las acciones del Estado en estas áreas y su permanencia. Es evidente que la Administración Pública debe entrar en lugares que han sido segregados históricamente, pero la formalización de estas partes de la ciudad siguen patrones que a menudo pueden profundizar las desigualdades. La propia permanencia indefinida de un modelo policial 
distinto puede caracterizar una violencia simbólica, por la posibilidad de fortalecer el estigma social, en lugar de promover y garantizar los derechos. Además, la violencia policial y la violencia de los narcotraficantes no pueden distinguirse en su producto final: la pérdida de la alteridad. En este sentido, la UPP parece ser más de lo mismo en relación con los ciudadanos y el Estado, por lo que las investigaciones sobre su propia relación de poder dinámico y asimétrico social entre los que caminan la línea y los desviados.

\section{REFERENCIAS}

BRUM, Mario. Favelas e remocionismos ontem e hoje: da Ditadura de 1964 aos Grandes Eventos. O Social em Questão, v. 29. Rio de Janeiro: 2013.

CUNHA, Neiva; MELLO, Marco Antonio. Novos conflitos na cidade: a UPP e o processo de urbanização na favela. Dilemas: Revista de Estudos de Conflito e Controle Social, v. 4. Rio de Janeiro: 2011.

CANO, Ignácio (org.). “Os donos do Morro”: Uma avaliação exploratória das Unidades de Polícia Pacificadora (UPPs) no Rio de Janeiro. Fórum Brasileiro de Segurança Pública: 2012.

MIRANDA, Ana Paula. Militarização e direitos humanos: gramáticas em disputa nas políticas de segurança pública no Rio de Janeiro/ Brasil. Fórum Sociológico, n. 25 / 2014.

MOURÃO, Barbara. UPPs: uma polícia de que gênero? Rio de Janeiro: Cesec/UCAM, 2013.

OLIVEIRA, Fabiana; SADEK, Maria; ABRAMOVAY, Pedro; NUÑEZ, Izabel. UPPs, Direitos e Justiça. 1. ed. Rio de Janeiro: FGV Editora, 2012.

SILVA, Jailson; FERNANDES, Fernando; BRAGA, Raquel. Grupos criminosos armados com domínio de território: reflexões sobre a territorialidade do crime na região metropolitana do Rio de Janeiro. In: JUSTIÇA GLOBAL BRASIL. Segurança, Tráfico e Milícias no Rio de Janeiro. Rio de Janeiro: Fundação Heinrich Boll, 2008.

SILVA DE OLIVEIRA, Lívio. Limites e potencialidades da participação civil na segurança pública: um estudo de caso sobre as equipes técnicas do Território de Paz no Guajuviras. Dissertação de Mestrado em Sociologia. Porto Alegre: PPGS-UFRGS, 2014. 
TAYLOR, Ian; WALtON, Paul; YOUNG, Jock. La Nueva Criminologia. Buenos Aires: Amorrotu ed.; 1990. 